\title{
Clinical Effects of Tramiprosate in APOE4/4 Homozygous Patients with Mild Alzheimer's Disease Suggest Disease Modification Potential
}

\author{
S. Abushakra', A. Porsteinsson ${ }^{2}$, P. Scheltens ${ }^{3}$, C. Sadowsky 4 , B. Vellas ${ }^{5}$, J. Cummings ${ }^{6}$, S. Gauthier $^{7}$,

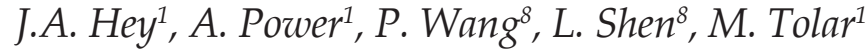

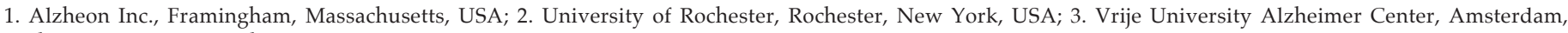

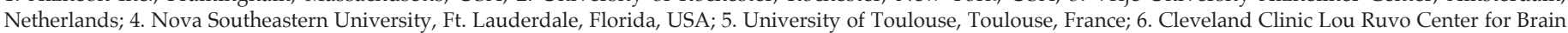
Health, Las Vegas, Nevada, USA; 7. McGill University and McGill Center for Studies in Aging, Montreal, Canada; 8. Pharmapace Inc., San Diego, California, USA

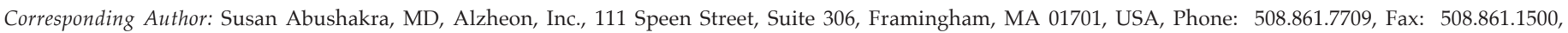
susan.abushakra@alzheon.com

J Prev Alz Dis 2017;4(3):149-156

Published online June 22, 2017, http:/ / dx.doi.org/10.14283/jpad.2017.26

\begin{abstract}
BACKGROUND: Alzheimer's Disease (AD) patients homozygous for the APOE4 allele (APOE4/4) have a distinct clinical and biological phenotype with high levels of beta amyloid $(A \beta)$ pathology and toxic $A \beta$ oligomers. Tramiprosate, an oral agent that inhibits $A \beta$ monomer aggregation into toxic oligomers, was evaluated in two Phase 3 Mild to Moderate AD studies which did not show efficacy in the overall population. Re-analyses of these trials showed the most consistent clinical benefits in APOE4/4 patients. We analyzed efficacy in the APOE4/ 4 patients with Mild disease.
\end{abstract}

OBJECTIVES: To determine the optimal stage of AD for future trials in APOE4/ 4 homozygotes.

DESIGN: Two randomized, double-blind, placebo-controlled parallel-arm multi-center studies of 78-weeks duration.

SETTING: Academic Alzheimer's disease centers, communitybased memory clinics, and neuropsychiatric research sites.

PARTICIPANTS: Participants included 2,025 AD patients with MMSE 16-26. Approximately 13-15\% had APOE4/4 genotype $(\mathrm{N}=147$ and 110 per study), mean age 71.1 years, 56\% females. Almost all were on stable symptomatic drugs.

INTERVENTION: Randomized subjects received oral placebo, $100 \mathrm{mg}$ BID, or 150mg BID of tramiprosate.

MEASUREMENTS: Co-primary outcomes were change from baseline in the ADAS-cog11 and CDR-SB. Disability assessment for dementia (DAD) was a secondary outcome.

RESULTS: In APOE4/4 homozygotes receiving 150mg BID tramiprosate, efficacy in the traditional Mild AD patients (MMSE 20-26) was higher than the overall group (MMSE 16-26) and efficacy in the Mild patients (MMSE 22-26) was highest. Tramiprosate benefits compared to placebo on ADAS-cog, CDR-SB, and DAD were $125 \%, 81 \%$ and $71 \%$, respectively $(\mathrm{p}<0.02)$. The Mild subgroup (MMSE 22-26) showed cognitive stabilization with no decline over 78 weeks, both ADAS-cog and DAD effects increased over time. Tramiprosate safety in APOE4/ 4 patients was favorable. Most common adverse events were nausea, vomiting, depression and decreased weight.

CONCLUSIONS: The Mild subgroup of APOE4/4 AD patients (MMSE 22-26) showed larger benefits on the high dose of tramiprosate than the overall Mild and Moderate group. Consistent with its preclinical effects on $\mathrm{A} \beta$ oligomers, tramiprosate seemed to stabilize cognitive performance, supporting its disease modification potential. Confirmatory studies using ALZ-801, an improved pro-drug formulation of tramiprosate, will target APOE4/4 patients with Mild AD.
Key words: Tramiprosate, Alzheimer's, APOE4, amyloid oligomers.

Abbreviations: AE: Adverse Event(s); AD: Alzheimer's Disease; A : Beta Amyloid; ADAS-cog: Alzheimer's Disease Assessment Scale-cognitive Subscale; APOE4 = Apolipoprotein E4; $\varepsilon 4$ allele of the apolipoprotein E gene; ARIA-E: Amyloid Related Imaging Abnormalities-Edema; CBL: Change from Baseline; CDR-SB: Clinical Dementia Rating Scale-Sum of Boxes; DAD: Disability Assessment for Dementia; EU: European; GI: Gastrointestinal; ITT: Intentto-Treat; LS: Least Squares; MedDRA: Medical Dictionary for Regulatory Activities; MMRM: Mixed Effects Model with Repeated Measures; MMSE: Mini-Mental State Examination; MRI: Magnetic Resonance Imaging; NA: North American; NPI: Neuropsychiatric Inventory; SAP: Statistical Analysis Plan; SAE: Serious Adverse Event(s); TEAE: Treatment-Emergent Adverse Event(s)

\section{Introduction}

The $\varepsilon 4$ allele of the apolipoprotein E gene (APOE4) is an important risk factor for Alzheimer's disease (AD), second only to age (1). The APOE4 genotype confers a 4 to 12 -fold higher risk of $\mathrm{AD}$ and lowers the age of onset by approximately $10-15$ years $(2,3)$. APOE carrier proteins, which play an important role in membrane maintenance, are produced by astrocytes in response to injury and regulate neuronal Beta amyloid $(\mathrm{A} \beta)$ metabolism $(4,5)$. The APOE4 isoform reduces clearance of $A \beta$ monomers, promotes their aggregation into soluble toxic oligomers and insoluble fibrils $(5,6)$, and may directly stimulate $A \beta$ synthesis (7). These effects on $A \beta$ are thought to underly the high risk of AD in APOE4 carriers. .

APOE4 carriers have a distinct biological and clinical phenotype across imaging, biomarker and clinical studies. APOE4 carriers have higher rates of positive amyloid scans across all stages of disease (8, 9), show greater cortical amyloid burden, regional hypometabolism (10), and more marked hippocampal atrophy than non-carriers (11). Their clinical profile reflects hippocampal deficits with delayed memory 
affected earlier than executive function or language (12, 13). APOE $4 / 4$ homozygotes exhibit significantly greater amyloid burden than heterozygotes (14). APOE4/4 homozygotes thus represent a biologically homogenous population of $\mathrm{AD}$ patients that is enriched for amyloid pathology.

Tramiprosate is an oral amyloid targeted agent that inhibits $A \beta$ oligomer formation and fibrillar (plaque) deposition in transgenic animal models (15-17). Tramiprosate was previously evaluated in two Phase 3 trials in Mild to Moderate AD. The North American trial results did not show efficacy in the overall study population (18), and the European trial was terminated before completion. Analyses of these studies based on the number of APOE4 alleles showed a gene-dose effect revealing the largest efficacy signals in APOE4/4 homozygotes, intermediate in APOE4 heterozygotes and lowest in APOE4 non-carriers (19). This parallels the rank order of amyloid positivity in APOE4 subgroups from recent $\mathrm{AD}$ trials, with highest rates $(\sim 98 \%)$ in APOE4/ 4 homozygotes, and lowest rates $(\sim 60 \%)$ in noncarriers (20). In APOE4/4 homozygotes, tramiprosate showed promising clinical benefits in the overall Mild to Moderate AD group (19). Since $A \beta$ toxicity is thought to play a larger role at the early stages of $\mathrm{AD}$, we explored the efficacy of tramiprosate in two Mild subgroups of APOE4/ 4 patients.

\section{Methods}

\section{Study Design}

The Phase 3 tramiprosate studies included a North American (NA) study (NCT00088673) and a European (EU) study (NCT00217763). The NA and EU study results were previously reported $(18,19)$. These studies were conducted in accordance with ICH guidance and all local regulations. Both the NA study (Study CL-758007) and EU study (CL-758010) were multi-center, randomized, placebo-controlled, double-blind, parallel-arm studies of 78 weeks duration. The EU study had similar design and outcome measures, but was terminated before completion when the NA study did not show significant efficacy in the overall study population.

\section{Study Participants}

Both studies enrolled AD patients with baseline Mini-Mental State Examination (MMSE) scores of 16-26, inclusive. The NA study included 67 centers in the US and Canada, and the EU study included centers from 11 Western European countries. The AD diagnosis was based on clinical criteria with brain MRI or CT imaging as supportive evidence, since amyloid imaging was not widely available at the time. Participants were enrolled and randomized into one of three arms (placebo, 100mg
BID and 150mg BID of tramiprosate) and received study drug for 78 weeks, with study visits occurring every 13 weeks. The NA study allowed treatment with cholinesterase inhibitors alone, or with memantine, at stable doses for at least 12 weeks. The EU study allowed cholinesterase inhibitors only, and prohibited memantine use.

\section{Outcome Measures}

Co-primary outcomes in both studies were changes from baseline (CBL) to Week 78 on the 11-item Alzheimer's Disease Assessment Scale-Cognitive Subscale (ADAS-cog) (21), and on the Clinical Dementia Rating - Sum of Boxes (CDR-SB) (22). The CDR-SB combines cognitive and functional measures. Secondary outcomes included the Disability Assessment for Dementia (DAD) (23) which evaluates function and disability; the MMSE (24) which is a cognitive staging instrument; and the 12-item Neuropsychiatric Inventory (NPI) (25) which evaluates the presence and severity of 12 neuropsychiatric symptoms that commonly occur in dementia patients. Higher values on the ADAS-cog, CDR and NPI indicated worsening, while higher values on the DAD and MMSE indicate improvement.

\section{Clinical Datasets: NA and EU Studies}

The NA and EU studies included 973 and 870 participants, respectively, with known genotype, of whom 147 and 110 were APOE4/4 homozygotes. In these APOE4/ 4 homozygous subgroups, efficacy and safety datasets were analyzed separately and as a pooled dataset (discussed below).

\section{Analyses by APOE4 Genotype}

APOE4 status was one of the pre-specified covariates in the statistical analysis plan (SAP). The APOE4 genotype-treatment interaction was found to be significant for both primary outcomes $(p<0.05)$. In addition, there was an overall significant treatment effect by APOE4 by visit interaction for ADAS-cog $(\mathrm{p}<$ 0.05 ). These results suggested that treatment effects are dependent on APOE4 status. Therefore, an analysis in APOE4 carriers and non-carriers was performed, and showed significant efficacy in APOE4 carriers. We recently further analyzed and published the efficacy results of both studies based on the number of APOE4 alleles (19). A subset of patients from both studies had MRI at baseline and end of study, the MRI safety analyses for vasogenic edema (ARIA-E) were recently reported (19). 


\section{Efficacy analyses}

Efficacy analyses in this report are for subjects in the intent-to-treat (ITT) population of each study, who had a baseline and at least one post-baseline assessment, received at least one dose of study drug, and carried the APOE4/ 4 genotype.

The mixed effect model with repeated measures (MMRM) was the analytical method specified in the prospectively developed SAP for the NA study. This SAP had undergone Food and Drug Administration review prior to database lock on March 30, 2007. This model included the following terms: treatment, visit, treatment by visit interaction, study site, and baseline value of the primary endpoint to be analyzed. The analyses were performed using this original MMRM. The SAP of the NA study included the following factors as covariates to be tested for interaction with drug effect: age, gender, use of symptomatic drugs, and APOE4 genotype. The SAP also required that efficacy results be summarized by APOE genotype.

An additional MMRM analysis termed 'updated MMRM' was performed that included baseline MMSE values in the model to be consistent with current practice (19). Both MMRM methods yielded similar results and conclusions. Results presented in this paper are from the updated MMRM analyses. Study sites recruiting few patients were pooled to avoid having a site with only one subject from a dose arm in an APOE4 subgroup.

The EU Phase 3 study was prematurely discontinued when the results of the NA study became available. Most patients had completed the Week 52 visit while approximately $30 \%$ had completed Week 78 evaluations. Therefore, the last two visits for subgroup analyses with each of the Mild subgroups had a small sample size and MMRM analysis was not suitable. The EU dataset was combined with the NA dataset and analyzed by MMRM, and is considered a supportive sensitivity analysis.

\section{Safety analyses}

The combined NA and EU (Pooled) safety population included all subjects who received at least one dose of study drug and who had the APOE4/4 genotype. Safety analyses included treatment-emergent adverse events (TEAE) and serious adverse event (SAE). Adverse events were coded using MedDRA version 9.0. An exploratory comparison of incidence of TEAE between placebo and the two active arms was performed using Fisher's exact test.

\section{Exploratory Analyses Based on Baseline Disease Severity}

Additional exploratory analyses were performed based on baseline disease severity, and evaluated efficacy on
ADAS-cog, CDR-SB, DAD, NPI and MMSE. Patients were classified by baseline MMSE category (16-26; 20-26; 22-26), and data analyzed using the same MMRM method as the primary analysis, with the exception that site was not included as a factor in the model due to the smaller number of patients per site in the Mild subgroups.

Since these APOE4 subgroup analyses are exploratory and are being used to inform the design of future studies, the p-values presented were not adjusted for multiple statistical comparisons. In this paper, the MMSE category of 20-26 is termed the 'traditional Mild $\mathrm{AD}^{\prime}$ group, and the MMSE category of 22-26 is termed 'Mild AD'.

\section{Exploratory Analyses of Divergence of Slopes between Dose Arms}

To explore the disease modification potential of tramiprosate, the difference in slopes between treatment arms was evaluated for statistical significance. An MMRM model was used for these analyses, with model terms including treatment, visit days as a numerical variable, baseline value of the response variable, baseline MMSE value, and treatment by visit-days interaction.

\section{Specification of Model Variables (MMRM)}

\section{Response Variables}

The primary outcome variables for all inferential statistical analyses were the changes from baseline (CBL) to each visit i for ADAS-cog and CDR-SB, as defined by:

$$
\begin{aligned}
& \left.\left.\mathrm{CBL}(\mathrm{ADAS}-\mathrm{cog})_{\text {Visit }}=\mathrm{ADAS}-\mathrm{cog}\right)_{\text {Visit } i}-\mathrm{ADAS}-\mathrm{cog}\right)_{\text {Baseline }} \\
& \mathrm{CBL}(\mathrm{CDR}-\mathrm{SB})_{\text {Visit } i}=(\mathrm{CDR}-\mathrm{SB})_{\text {Visit } i}-(\mathrm{CDR}-\mathrm{SB})_{\text {Baseline }}
\end{aligned}
$$

\section{Independent Variables}

The treatment group was regarded as a class variable with three levels: (1) tramiprosate $150 \mathrm{mg}$ BID, (2) tramiprosate $100 \mathrm{mg}$ BID, and (3) placebo. The visit variable was included as a class variable with values standing for the visit numbers (Visit 5 to Visit 10, corresponding to Weeks 13 to 78), and was used to index the within-subject observations over time.

\section{Covariates}

The baseline value of the dependent variable was entered as a continuous covariate since both absolute and percent changes over time in the outcome measure correlate with its value at the baseline visit. Baseline MMSE score was used as a measure of AD severity at baseline and was entered as a continuous covariate. The autoregressive order 1 covariance structure was used to model the within-subject correlation across the different visits. 


\begin{tabular}{|c|c|c|c|c|c|c|}
\hline \multirow[b]{2}{*}{ Demographic } & \multicolumn{3}{|c|}{ Study CL-758007 (NA) } & \multicolumn{3}{|c|}{ Study CL-758010 (EU) } \\
\hline & $\begin{array}{c}\text { Placebo } \\
\mathrm{N}=58\end{array}$ & $\begin{array}{c}100 \mathrm{mg} \text { BID } \\
\mathrm{N}=48\end{array}$ & $\begin{array}{c}150 \mathrm{mg} \text { BID } \\
\mathrm{N}=41\end{array}$ & $\begin{array}{c}\text { Placebo } \\
\mathrm{N}=38\end{array}$ & $\begin{array}{c}100 \mathrm{mg} \text { BID } \\
\mathrm{N}=38\end{array}$ & $\begin{array}{c}150 \mathrm{mg} \text { BID } \\
\mathrm{N}=34\end{array}$ \\
\hline Age, y, mean (SD) & $72.6(7.48)$ & $71.7(7.04)$ & $71.3(8.08)$ & $70.1(6.29)$ & $69.7(8.29)$ & $70.3(6.42)$ \\
\hline Female, n (\%) & $34(58.6)$ & $26(54.2)$ & $23(56.1)$ & $20(52.6)$ & $23(60.5)$ & $18(52.9)$ \\
\hline Race, n (\% White) & $57(98.3)$ & $47(97.9)$ & $39(95.1)$ & $38(100)$ & $38(100)$ & $34(100)$ \\
\hline Education, mean years (SD) & $14.4(3.0)$ & $15.1(4.14)$ & $13.8(3.06)$ & $10.2(3.57)$ & $11.2(4.43)$ & $10.8(4.39)$ \\
\hline BMI, mean $\mathrm{kg} / \mathrm{m}^{2}(\mathrm{SD})$ & $24.89(4.22)$ & $24.91(4.44)$ & $26.19(3.8)$ & $24.74(3.63)$ & $25.72(4.4)$ & $25.36(3.32)$ \\
\hline \multicolumn{7}{|c|}{ Baseline Clinical Characteristics, $n(\%)$} \\
\hline Subjects taking AChEIs & $58(100)$ & $47(97.9)$ & $40(97.6)$ & $38(100)$ & $38(100)$ & $33(97.1)$ \\
\hline Subjects taking Memantine & $26(44.8)$ & $21(43.8)$ & $23(56.1)$ & $0(0)$ & $0(0)$ & $0(0)$ \\
\hline \multicolumn{7}{|c|}{ Baseline Efficacy Measures, Mean (SD) } \\
\hline ADAS-cog & $21.7(6.86)$ & $23.1(8.64)$ & $22.4(7.05)$ & $22.6(8.13)$ & $21.8(8.65)$ & $20.3(6.29)$ \\
\hline CDR-SB & $5.9(3.1)$ & $5.4(2.65)$ & $6.0(2.48)$ & $6.3(2.91)$ & $5.7(2.86)$ & $5.0(2.49)$ \\
\hline DAD & $75.2(22.43)$ & 77.9 (19.35) & 75.8 (20.08) & 71.3 (21.98) & 76.1 (15.81) & $81.7(18.43)$ \\
\hline NPI & $8.4(9.26)$ & $8.9(10.86)$ & $9.2(10.98)$ & $10.1(9.91)$ & $10.3(9.91)$ & $8.1(7.46)$ \\
\hline MMSE & $21.1(3.12)$ & $20.4(3.54)$ & $21.1(3.33)$ & $20.7(2.92)$ & $21.8(2.84)$ & $21.9(2.96)$ \\
\hline
\end{tabular}

Higher values for ADAS-cog, CDR-SB and NPI indicate greater disease severity, and lower values in DAD and MMSE indicate greater severity. APOE4 = apolipoprotein E4; AChEIs = acetylcholinesterase inhibitors; MMSE = Mini Mental State Examination; ADAS-cog = Alzheimer's Disease Assessment Scale-cognitive subscale; CDR-SB = Clinical Dementia Rating Sum of Boxes; DAD = Disability Assessment for Dementia; NPI = Neuropsychiatric Inventory.

\section{Results}

\section{Demographics and Baseline Characteristics}

The NA and EU studies enrolled a total of 1052 and 973 patients, respectively. The ITT population from the NA and EU studies with known APOE genotypes consisted of 973 and 870 patients, of whom 147 and 110 were APOE4/ 4 homozygotes, respectively. The demographics of APOE4 non-carriers, heterozygotes and homozygotes were balanced except for a younger mean age in APOE4/ 4 homozygotes (19).

Demographics and baseline scores of the APOE4/4 homozygous subgroups in each study are shown in Table 1 . In the NA study, the mean age was 72 years, 57\% were female, and 97\% were caucasian. In the EU study, the mean age was 70 years, $56 \%$ were female, and $100 \%$ were Caucasian. In both studies $~ 99 \%$ of subjects were on cholinesterase inhibitors. The NA study had $\sim 48 \%$ of patients taking memantine, while the EU study did not allow memantine use. APOE4/4 subgroups in each study showed similar patient demographics across all three dose arms.

Baseline clinical scores in the NA and EU studies were respectively: MMSE 20.9 and 21.5; ADAS- $\operatorname{cog} 22.3$ and 21.6; CDR-SB 5.8 and 5.7; DAD 76.3 and 76.1; and NPI 8.8 and 9.5. In the NA study the low dose arm showed higher ADAS-cog but lower CDR-SB baseline scores than the other two arms. In the EU study the placebo showed higher ADAS-cog and CDR-SB scores than the active dose arms.

\section{Efficacy}

\section{Efficacy Outcomes in the APOE4/4 Subgroup by Baseline MMSE Range- NA study}

In Table 2 the effects of both tramiprosate doses (100mg BID and 150mg BID) on the co-primary outcomes ADAS-cog and CDR-SB are shown for the last two visits (Weeks 65 and 78) for the NA Study. The overall group includes Mild and Moderate subjects (MMSE 16-26), while the two Mild groups include subjects with MMSE 20-26 and 22-26 (Table 2). At the high dose the effects on both ADAS-cog and CDR-SB increase progressively and become more significant as the MMSE range is limited to the traditional Mild group (20 and above), and then to the Mild group (22 and above).

The time course of tramiprosate effects on ADAS-cog, CDR-SB and DAD is shown at each visit in Figure 1. In the Mild group (MMSE 22 and above) at the high dose, cognitive scores improve and remain above baseline for 78 weeks; CDR-SB also remains above baseline for 65 weeks and shows a small decline compared to baseline at 78 weeks. In this Mild group the DAD also shows increasing benefit with time and reaches significance at 78 weeks. Due to this observation of divergent treatment effects between the $150 \mathrm{mg}$ and placebo groups, an additional analysis was performed to examine the difference in the slopes of these two arms. The difference in slopes between placebo and the high dose arm was significant for ADAS-cog $(\mathrm{p}=0.015)$, non-significant for 
Table 2. Tramiprosate Effects on Co-Primary Outcomes in APOE4/4 Homozygotes: Mild/Moderate and Mild Subgroups (NA Study)

\begin{tabular}{|c|c|c|c|c|c|c|c|c|c|c|c|c|c|}
\hline \multirow[t]{3}{*}{ MMSE Group } & \multirow[t]{3}{*}{ Week } & \multicolumn{12}{|c|}{ LS Mean Difference in CBL } \\
\hline & & \multicolumn{6}{|c|}{ 100mg BID } & \multicolumn{6}{|c|}{$150 \mathrm{mg}$ BID } \\
\hline & & \multicolumn{3}{|c|}{ ADAS-cog } & \multicolumn{3}{|c|}{ CDR-SB } & \multicolumn{3}{|c|}{ ADAS-cog } & \multicolumn{3}{|c|}{ CDR-SB } \\
\hline Overall & 65 & 37 & $-1.83(1.22)$ & 0.13 & 37 & $0.18(0.41)$ & 0.67 & 34 & $-3.55(1.26)$ & 0.0050 & 34 & $-0.81(0.42)$ & 0.0586 \\
\hline $16-26$ & 78 & 36 & $-0.83(1.24)$ & 0.50 & 36 & $0.24(0.42)$ & 0.57 & 34 & $-2.69(1.27)$ & 0.0351 & 34 & $-0.56(0.43)$ & 0.19 \\
\hline $20-26$ & 78 & 23 & $-1.94(1.34)$ & 0.15 & 23 & $-0.42(0.43)$ & 0.33 & 23 & $-3.80(1.34)$ & 0.0048 & 23 & $-0.83(0.43)$ & 0.0577 \\
\hline Mild & 65 & 17 & $-1.70(1.48)$ & 0.25 & 17 & $-0.64(0.48)$ & 0.19 & 18 & $-4.51(1.45)$ & 0.0021 & 18 & $-1.59(0.48)$ & 0.0011 \\
\hline $22-26$ & 78 & 17 & $-2.49(1.49)$ & 0.0958 & 17 & $-0.35(0.49)$ & 0.48 & 18 & $-5.66(1.46)$ & 0.0001 & 18 & $-1.14(0.48)$ & 0.0197 \\
\hline
\end{tabular}

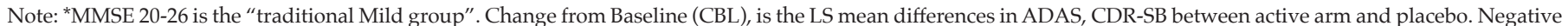

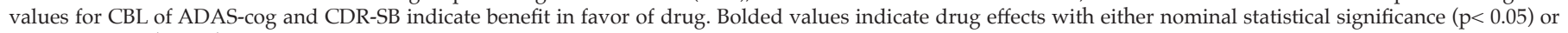
positive trends $(\mathrm{p}<0.1)$.

Table 3. Effects of Tramiprosate 150mg BID in APOE4/ 4 Patients with Mild AD (MMSE 22-26, NA Study): Percent Drug Benefit Compared to Placebo

\begin{tabular}{|c|c|c|c|c|c|}
\hline \multirow{2}{*}{$\begin{array}{l}\text { Outcome } \\
\text { Measures }\end{array}$} & \multirow[b]{2}{*}{$\begin{array}{l}\text { CBL to } \\
\text { Week }\end{array}$} & \multicolumn{4}{|c|}{ MILD GROUP (MMSE 22-26) } \\
\hline & & $\mathbf{N}$ & $\begin{array}{c}\text { Place- } \\
\text { bo-Cor- } \\
\text { rected LS } \\
\text { Mean (SE) }\end{array}$ & $P$ value & $\begin{array}{l}\% \text { Drug } \\
\text { Benefit }^{*}\end{array}$ \\
\hline \multirow[t]{3}{*}{ ADAS-cog } & 52 & 18 & $-3.92(1.43)$ & 0.007 & $185 \%$ \\
\hline & 65 & 18 & $-4.51(1.45)$ & 0.002 & $170 \%$ \\
\hline & 78 & 18 & $-5.66(1.46)$ & 0.0001 & $125 \%$ \\
\hline \multirow[t]{3}{*}{ CDR-SB } & 52 & 18 & $-1.00(0.47)$ & 0.036 & $126 \%$ \\
\hline & 65 & 18 & $-1.59(0.48)$ & 0.001 & $130 \%$ \\
\hline & 78 & 18 & $-1.14(0.48)$ & 0.0197 & $81 \%$ \\
\hline \multirow[t]{3}{*}{ DAD } & 26 & 21 & $4.03(3.74)$ & 0.284 & $122 \%$ \\
\hline & 52 & 18 & 5.49 (3.93) & 0.165 & $62 \%$ \\
\hline & 78 & 18 & $11.39(4.03)$ & 0.006 & $71 \%$ \\
\hline \multirow[t]{3}{*}{ NPI } & 26 & 21 & $-2.49(2.93)$ & 0.396 & $213 \%$ \\
\hline & 52 & 18 & $-3.73(3.09)$ & 0.2298 & $143 \%$ \\
\hline & 78 & 18 & $-1.93(3.14)$ & 0.5396 & $53 \%$ \\
\hline \multirow[t]{3}{*}{ MMSE } & 26 & 21 & $-0.37(0.80)$ & 0.648 & $-48 \%$ \\
\hline & 52 & 18 & $0.32(0.83)$ & 0.7035 & $34 \%$ \\
\hline & 78 & 18 & $0.47(0.85)$ & 0.585 & $24 \%$ \\
\hline
\end{tabular}

*\% Drug benefit is indicated as positive when drug is better than placebo. For ADAS-cog, CDR-SB, and NPI negative differences indicate drug benefit, for DAD and MMSE positive differences indicate drug benefit. Bolded values indicate either statistical significance (nominal $p<0.05$ ) or positive trends $(p<0.1)$. CBL: Change from Baseline.

CDR-SB $(p=0.114)$, and showed a positive trend for DAD $(\mathrm{p}=0.068)$.

The percentage benefit compared to placebo in the Mild subgroup (MMSE 22-26) that showed the largest effects is shown in Table 3. For ADAS-cog and CDR-SB, and for the secondary outcomes of DAD, NPI and MMSE, results are shown from the last three visits at which they were assessed. In AD trials a benefit of at least 25\% compared to placebo is considered the minimal clinically relevant benefit. The percentage benefits on ADAS-cog, CDR-SB and DAD $(125 \%, 81 \%$ and $71 \%$, respectively) are well above that threshold. For NPI and MMSE the percentage benefit of $53 \%$ and $24 \%$, respectively, was not statistically significant, but was directionally positive.

In the Mild group (MMSE 22-26) at the 78-Week endpoint, the effect size (Cohen's d) and 95\% confidence intervals (CI) were as follows: for ADAS-cog -0.54 (Lower CI: -0.82; Upper CI: -0.25); for CDR-SB -0.39 (Lower CI: -0.71; Upper CI: -0.06); and for DAD 0.52 (Lower CI: 0.15; Upper CI: 0.89).

\section{Efficacy Outcomes in the APOE4/4 Subgroup by Baseline MMSE Range- Combined Studies}

In the combined datasets, the time course of tramiprosate effects on ADAS-cog, CDR-SB and DAD is shown in Figure 2, for the overall (Mild and Moderate group) and Mild group (MMSE 22-26). For the high dose least squares (LS) mean differences in the overall group at 78 weeks on ADAS-cog, CDR-SB and DAD were -2.3, $\mathrm{p}=0.04 ;-0.4, \mathrm{p}=0.26$; and $-1.8, \mathrm{p}=0.49$, respectively; and in the Mild group were $-4.8, \mathrm{p}=0.001 ;-0.9, \mathrm{p}=0.05 ; 8.4$, $\mathrm{p}=0.02$, respectively. The effects of the high dose on all three outcomes at the study endpoint were again higher in the Mild group and achieved nominal significance.

\section{Safety}

Across the two studies there were 263 APOE4/4 patients in the safety population. The most common TEAE are shown in Table 4. The nature of adverse events in the APOE4/4 group is similar to the overall study population and there were no events of ARIA-E on active drug (19). The incidence of nausea, vomiting, depression and weight loss was higher in the high dose arm than placebo, but was not statistically significant (nominal p-value $>0.05$ ). The majority of nausea and vomiting 
Figure 1. Time Course of Effect in APOE4 / 4 Homozygotes in the Overall and Mild Subgroup (MMSE 22-26, NA Study)
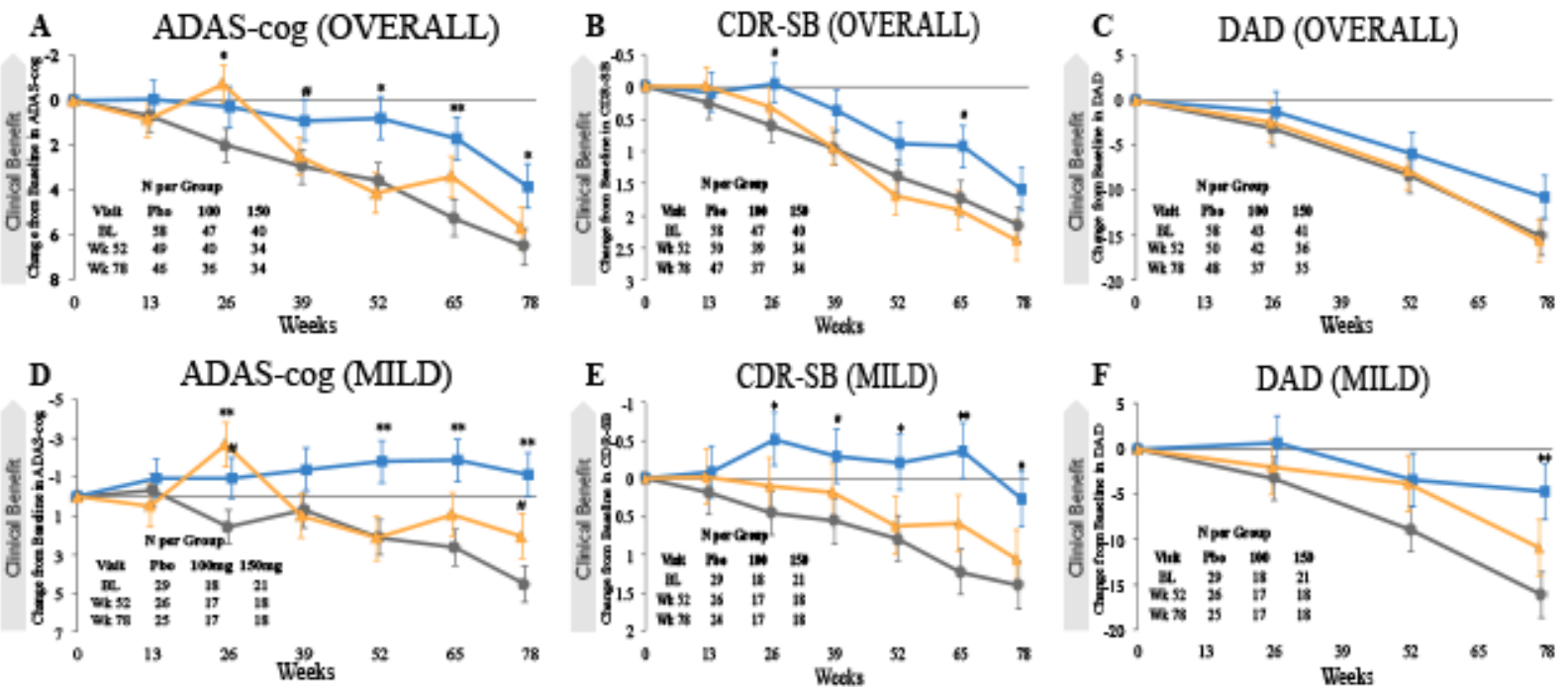

- Placebo $-100 \mathrm{mg} \mathrm{BID}-150 \mathrm{mg} \mathrm{BD}$

CBL determined by MMRM analysis, error bars are SEM. For ADAS-cog and CDR-SB, negative CBL indicate
improvement. DAD was performed every 26 weeks only. \# indicates $\mathrm{p}<0.1$; ${ }^{*}$ indicates $\mathrm{p}<0.05$; ${ }^{*}$ indicates $\mathrm{p}<0.01$.

Figure 2. Time Course of Effect in APOE4/ 4 Homozygotes in the Overall and Mild Subgroup (MMSE 22-26, Combined Studies)

ADAS-cog (OVERALL)

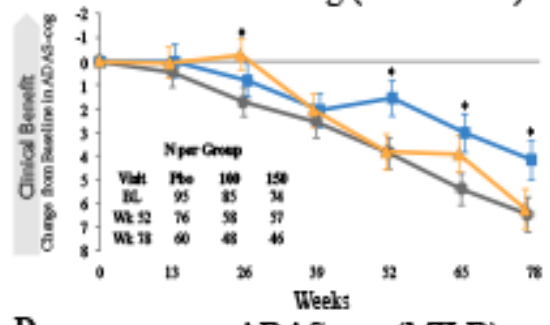

D

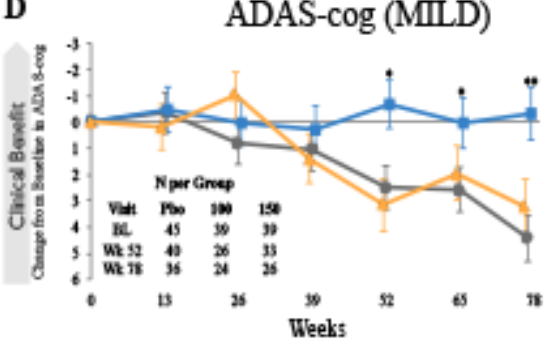

CDR-SB (OVERALL)

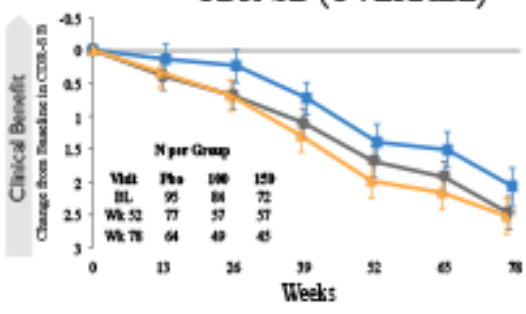

CDR-SB (MILD)

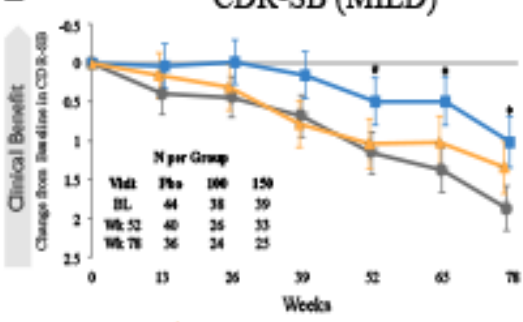

DAD (OVERALL)

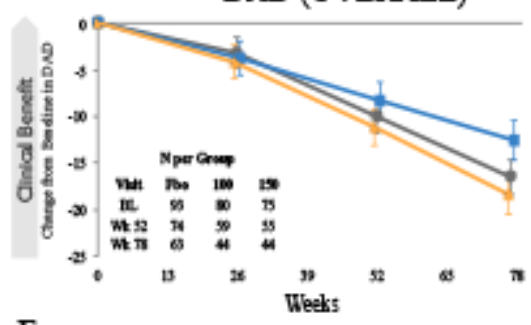

DAD (MILD)

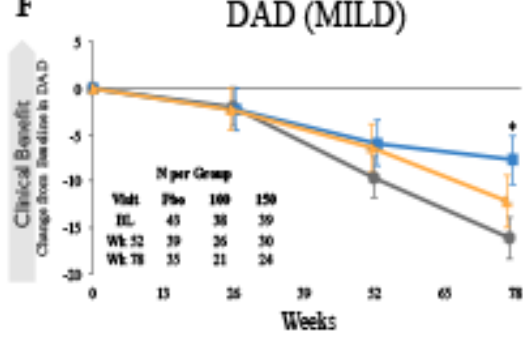

Placebo $-100 \mathrm{mg} \mathrm{BID}-150 \mathrm{mg} \mathrm{BID}$

CBL determined by MMRM analysis, error bars are SEM. For ADAS-cog and CDR-SB, negative CBL indicate improvement, for DAD positive CBL indicates improvement. DAD was performed every 26 weeks only. \# indicates $\mathrm{p}<0.1$; ${ }^{*} \mathrm{p}<0.05 ;{ }^{* *} \mathrm{p}<0.01$.

events were mild or moderate and less than five percent led to discontinuation. The incidence of serious AE was lower in active arms ( $14 \%$ low dose, $11 \%$ high dose) than placebo $(22 \%)$. The incidence of headache was lower in the high dose $(1 \%)$ arm than placebo $(11 \%$, nominal p-value $=0.013)$.

\section{Discussion}

In APOE4/ 4 homozygotes the sensitivity analysis of tramiprosate efficacy based on disease stage suggests that the Mild subgroup with MMSE 22 and above is more likely to have larger and more sustained benefits compared to the overall Mild and Moderate group. Based on both the NA and NA/EU datasets, the ADAScog drug-placebo differences increase with time, and cognitive scores remain stable for 78 weeks (Figures 1D, 2D). The CDR-SB effect is also sustained at more than one point clinical benefit over placebo at 78 weeks (Figure $1 \mathrm{E})$. In both datasets, the differences in disability (DAD) also increase with time (Figures $1 \mathrm{~F}$ and $2 \mathrm{~F}$ ). The low tramiprosate dose in the Mild subgroup shows smaller 
and less consistent numerical benefits. At the high dose, the ADAS-cog divergence of slopes was statistically significant, and the DAD showed a positive trend.

Table 4. APOE4/ 4 Homozygotes: TEAE with incidence $>5 \%$ in overall group* (Combined NA and EU Safety Population)

\begin{tabular}{|l|c|c|c|}
\hline & Placebo & $\begin{array}{c}\text { Tramiprosate } \\
\text { 100mg BID }\end{array}$ & $\begin{array}{c}\text { Tramiprosate } \\
\text { 150mg BID }\end{array}$ \\
\hline Adverse Event, $\mathbf{n}(\%)$ & $\mathbf{N = 9 8}$ & $\mathbf{N = 8 6}$ & $\mathbf{N = 7 9}$ \\
\hline Nausea & $13(13)$ & $17(20)$ & $18(23)$ \\
\hline Vomiting & $7(7)$ & $9(11)$ & $11(14)$ \\
\hline Depression & $9(9)$ & $5(6)$ & $10(13)$ \\
\hline Decreased Weight & $4(4)$ & $3(4)$ & $9(11)$ \\
\hline Fall & $9(9)$ & $7(8)$ & $8(10)$ \\
\hline Back Pain & $3(3)$ & $4(5)$ & $7(9)$ \\
\hline Diarrhea & $8(8)$ & $8(9)$ & $6(8)$ \\
\hline Dizziness & $7(7)$ & $9(11)$ & $3(4)$ \\
\hline Headache* & $11(11)$ & $7(8)$ & $1(1)$ \\
\hline
\end{tabular}

TEAE with incidence $>5 \%$ in the 3 combined dose arms, and higher incidence in an active arm than placebo, are listed by descending frequency in the high dose arm. The incidence of nausea, vomiting, depression and decreased weight were not significantly higher in active dose arms than placebo $(p>0.05)$. *Note: Headache is included since incidence in the high dose arm was significantly lower than placebo $\mathrm{p}=0.013$.

The magnitude of benefits over placebo on ADAS-cog, CDR-SB, and DAD $(125 \%, 81 \%$ and $71 \%$, respectively) was large and well above the $25 \%$ threshold that is considered the minimum clinically meaningful difference by clinical and regulatory experts. This is supported by the effect sizes at 78-Week endpoint being $>0.5$ for ADAS-cog and DAD, and 0.4 for CDR-SB.

$\mathrm{AD}$ patients who are $\mathrm{APOE} 4 / 4$ homozygotes represent a population with a significant burden of amyloid pathology including oligomers, and this may explain their reported preferential response to tramiprosate, which inhibits assembly of $A \beta$ oligomers $(6,17)$. The improved clinical response in the Mild AD subgroups is also consistent with preclinical effects of tramiprosate on $A \beta$ aggregation (17), since soluble $A \beta$ oligomers are thought to play an important and early role in synaptic toxicity and neurodegeneration in $\operatorname{AD}(26,27)$. This is supported by Amyloid imaging data from clinical trials showing ongoing plaque deposition in APOE4 carriers at the Mild but not the Moderate stage of disease (28).

The magnitude of the tramiprosate effect on CDR-SB in this analysis is consistent with the efficacy signal from a study in Early/Mild AD with the anti-amyloid antibody aducanumab (29). However, the efficacious doses of aducanumab in the latter study were associated with a high incidence of vasogenic edema/ARIA-E in APOE4 carriers; even with a dose titration regimen the incidence of ARIA-E was 35\% (30).

The main limitation of the current efficacy analyses is that they are post-hoc subgroup analyses from the NA study that did not achieve its primary objectives. The sample size of the APOE4/4 dataset was also limited, especially in the Mild subgroups. Within the APOE4/4 homozygous patients, randomization was not stratified based on baseline severity and the three dose arms were not precisely comparable, although the MMRM analysis included baseline severity in the model. Therefore, these findings require confirmation in prospectively defined studies in the APOE4/4 homozygous patients at the Mild AD stage (MMSE 22 and above).

From the safety perspective, tramiprosate showed a favorable safety profile in APOE4/4 homozygotes, with a similar profile to the other groups. The most common gastrointestinal events of nausea and vomiting are most likely mediated by a direct, local gastrointestinal (GI) irritation effect of tramiprosate in some subjects. This GI tolerability is being addressed by development of ALZ-801, a new pro-drug formulation (31). ALZ801 has shown substantially improved GI tolerability as well as more consistent plasma levels of tramiprosate in Phase $1 \mathrm{~b}$ studies in over 160 healthy elderly subjects (31). For future studies, ALZ-801 will be used at a dose that provides bioequivalent exposures to tramiprosate $150 \mathrm{mg}$ BID. Regarding the risk of vasogenic edema, MRI analyses from the two Phase 3 studies showed no evidence of vasogenic edema/ARIA-E with tramiprosate (19), even in the high risk group of APOE4 carriers (29).

\section{Conclusions}

Reanalysis of two prior tramiprosate studies showed promising efficacy in the APOE4/4 homozygous patients with Mild and Moderate AD. In this sensitivity analysis, the subgroup of Mild AD patients (MMSE 22 and above) shows larger and more sustained clinical effects on the high dose of tramiprosate. The cognitive scores improve and remain above baseline throughout the 78 weeks of treatment. The divergence of slopes (non-parallel slopes) between the two arms was significant. These effects on ADAS-cog were associated with significant and sustained benefits on function and disability over the study duration. This overall efficacy profile is supportive of a potential disease modifying effect and is consistent with the molecular mechanism of tramiprosate, inhibiting formation of toxic $A \beta$ oligomers. The safety profile in the overall APOE4/4 homozygous group was favorable. Prospective confirmation of these clinical effects in APOE4/4 homozygotes at the Mild stage of $\mathrm{AD}$ is warranted. The focus on this genetically-defined population represents a precision medicine approach to $\mathrm{AD}$ that has been successful in other challenging diseases.

Acknowledgements: We thank the investigators, their staff, and the individuals with AD who participated in the trials and their caregivers. We thank Dr. Sandra Saouaf and Christine Rathbun for editorial assistance. We thank our scientific advisors for their valuable review and commentary.

Funding: Alzheon Inc. funded the above analyses and manuscript preparation. Bellus Health (previously Neurochem), the original sponsor, had funded the 
tramiprosate Phase 3 studies.

Conflict of interest: Drs. Abushakra, Hey, Power and Tolar are employees of Alzheon, Inc. and hold stock or stock options of Alzheon, Inc. Drs. Porsteinsson, Scheltens, Sadowsky, Cummings, Gauthier, Vellas were investigators in the tramiprosate Phase 3 program and serve as advisors to Alzheon, Inc., and receive advisory fees and/or stock options of Alzheon. Drs. Shen and Mr. Wang serve as statistical consultants to Alzheon.

Ethical standards: The study protocols were approved by local ethics committees, and were conducted in accordance with ICH standards and all local regulations.

\section{References}

1. Roses AD. Apolipoprotein E alleles as risk factors in Alzheimer's disease. Annu Rev Med. 1996; 47:387-400.

2. Breitner JC, Wyse BW, Anthony JC, et al. APOE-epsilon4 count predicts age when prevalence of $\mathrm{AD}$ increases, then declines: the Cache County Study. Neurology. 1999; 53:321-331.

3. Raber J, Huang Y, Ashford JW. ApoE genotype accounts for the vast majority of AD risk and AD pathology. Neurobiol Aging. 2004; 25:641-650.

4. Mahley RW, Huang Y. Apolipoprotein E Sets the Stage: Response to Injury Triggers Neuropathology. Neuron. 2012; 76:871-885.

5. Holtzman DM, Herz J, Bu G. Apolipoprotein E and Apolipoprotein E Receptors: Normal Biology and Roles in Alzheimer Disease. Cold Spring Harb Perspect Med. 2012; 2:a006312.

6. Hashimoto T, Serrano-Pozo A, Hori Y, et al. Apolipoprotein E, especially apolipoprotein E4, increases the oligomerization of amyloid $\beta$ peptide. J Neurosci. 2012; 32:15181-15192.

7. Huang YA, Zhou B, Wernig M, Sudhof TC. ApoE2, ApoE3, and ApoE4 Differentially Stimulate APP Transcription and A $\beta$ Secretion. Cell. 2017; 168:427-441 e421.

8. Jansen WJ, Ossenkoppele R, Knol DL, et al. Prevalence of cerebral amyloid pathology in persons without dementia: a meta-analysis. JAMA. 2015 313:1924-1938.

9. Ossenkoppele R, Jansen WJ, Rabinovici GD, et al. Prevalence of amyloid PET positivity in dementia syndromes: a meta-analysis. JAMA. 2015; 313:19391949.

10. Ossenkoppele R, van der Flier WM, Zwan MD, et al. Differential effect of APOE genotype on amyloid load and glucose metabolism in AD dementia. Neurology. 2013; 80:359-365.

11. Liu Y, Yu JT, Wang HF, et al. APOE genotype and neuroimaging markers of Alzheimer's disease: systematic review and meta-analysis. J Neurol Neurosurg Psychiatry. 2015; 86:127-134.

12. Caselli RJ, Dueck AC, Osborne D, et al. Longitudinal modeling of age-related memory decline and the APOE epsilon4 effect. N Engl J Med. 2009; 361:255263.

13. Whitehair DC, Sherzai A, Emond J, et al. Influence of apolipoprotein E $\varepsilon 4$ on rates of cognitive and functional decline in mild cognitive impairment. Alzheimers Dement. 2010; 6:412-419.
14. Morris JC, Roe CM, Xiong C, et al. APOE predicts amyloid-beta but not tau Alzheimer pathology in cognitively normal aging. Ann Neurol. 2010; 67:122131.

15. Gervais F, Paquette J, Morissette C, et al. Targeting soluble Abeta peptide with Tramiprosate for the treatment of brain amyloidosis. Neurobiol Aging. 2007; 28:537-547.

16. Martineau E, de Guzman JM, Rodionova L, Kong X, Mayer PM, Aman AM. Investigation of the noncovalent interactions between anti-amyloid agents and amyloid beta peptides by ESI-MS. J Am Soc Mass Spectrom. 2010; 21:1506-1514

17. Kocis $\mathrm{P}$, Tolar M, Yu J, et al. Tramiprosate, an Anti-Amyloid Agent for the Treatment of Alzheimer's Disease, Inhibits Aß42 Oligomerization via a Novel Enveloping Mechanism. CNS Drugs. 2017. doi:10.1007/s40263-017-0434-z.

18. Aisen PS, Gauthier S, Ferris SH, et al. Tramiprosate in mild-to-moderate Alzheimer's disease - a randomized, double-blind, placebo-controlled, multicentre study (the Alphase Study). Arch Med Sci. 2011; 7:102-111.

19. Abushakra S, Porsteinsson A, Vellas B, et al. Clinical benefits of tramiprosate in Alzheimer's disease are associated with higher number of Apoe4 alleles: the "Apoe4 gene-dose effect". J Prev Alz Dis. 2016; 3(4):219-228.

20. Degenhardt EK, Witte MM, Case MG, et al. Florbetapir F18 PET Amyloid Neuroimaging and Characteristics in Patients With Mild and Moderate Alzheimer Dementia. Psychosomatics. 2016; 57:208-216.

21. Mohs RC, Cohen L. Alzheimer's Disease Assessment Scale (ADAS) Psychopharmacol Bull. 1988; 24:627-628.

22. Morris JC. The Clinical Dementia Rating (CDR): current version and scoring rules. Neurology. 1993; 43:2412-2414.

23. Gelinas I, Gauthier L, McIntyre M, Gauthier S. Development of a functional measure for persons with Alzheimer's disease: the disability assessment for dementia. Am J Occup Ther. 1999; 53:471-481.

24. Folstein MF, Folstein SE, McHugh PR. «Mini-mental state». A practical method for grading the cognitive state of patients for the clinician. J Psychiatr Res. $1975 ; 12: 189-198$

25. Cummings JL. The Neuropsychiatric Inventory: assessing psychopathology in dementia patients. Neurology. 1997; 48:S10-16.

26. Selkoe DJ, Hardy J. The amyloid hypothesis of Alzheimer's disease at 25 years. EMBO Mol Med. 2016; 8:595-608

27. Viola KL, Klein WL. Amyloid beta oligomers in Alzheimer's disease pathogenesis, treatment, and diagnosis. Acta Neuropathol. 2015; 129:183-206.

28. Liu E, Schmidt ME, Margolin R, et al. Amyloid-beta 11C-PiB-PET imaging results from 2 randomized bapineuzumab phase 3 AD trials. Neurology. 2015; 85:692-700.

29. Sevigny J, Chiao P, Bussiere $\mathrm{T}$, et al. The antibody aducanumab reduces Abeta plaques in Alzheimer's disease. Nature. 2016; 537:50-56.

30. Viglietta V, O'Gorman J, Williams L, et al. Aducanumab titration dosing regimen: 12-month interim analysis from prime, a randomized, doubleblind, placebo-controlled phase $1 \mathrm{~b}$ study in patients with prodromal or mild Alzheimer's disease. J Prev Alz Dis. 2016; 3:278.

31. Hey J, Abushakra S, Power A, Yu J, Versavel M, Tolar M. Phase development of Alz-801, a novel beta amyloid anti-aggregation prodrug of tramiprosate with improved drug properties, supporting bridging to the phase 3 program. Alzheimer's \& Dementia: The Journal of the Alzheimer's Association. 2016; 12:P613. 